\title{
Diplopia following Extraoral Maxillary Nerve Blockade for Trigeminal Neuralgia
}

\author{
Siddharth Chavali ${ }^{1}$ Girija P. Rath ${ }^{1} \quad$ Parmod K. Bithal ${ }^{2}$ \\ ${ }^{1}$ Department of Neuroanaesthesiology and Critical Care, \\ Neurosciences Centre, All India Institute of Medical Sciences \\ (AlIMS), New Delhi, India \\ 2 Department of Anesthesia and OR Administration, King Fahd \\ Address for correspondence Girija P. Rath, MD, DM, \\ Department of Neuroanaesthesiology and Critical Care, \\ Neurosciences Centre, All India Institute of Medical Sciences, \\ New Delhi 110029, India (e-mail: girijarath@yahoo.co.in).
} Medical City, Riyadh, Saudi Arabia

J Neuroanaesthesiol Crit Care 2018;5:193-194
Abstract
Keywords
- maxillary nerve block
- extraoral approach
- neurolytic agent
- complication
- abducens nerve palsy

We report a case of diplopia due to reversible abducens nerve block associated with extraoral maxillary blockade of the trigeminal nerve. This complication occurs despite precautions such as aspiration and confirmation of needle tip position with nerve stimulation. Knowledge of this condition and its potential cause should alert the physician to the importance of appropriate injection technique and an understanding of the management protocol.

\section{Introduction}

Blockade of branches of the trigeminal nerve distal to the Gasserian ganglion is a well-established procedure in the management of trigeminal neuralgia (TGN). Maxillary nerve (V2 division) block with alcohol is commonly performed when the maxillary distribution is predominantly affected. This technique has an acceptable safety profile, and hence performed as an outpatient procedure. The reported complications of maxillary nerve blockade are less than $1 \%$, and those described in the literature are dysesthesia, hypoesthesia, vertigo, and facial swelling. Distant complications, such as ipsilateral ocular disturbances, are extremely rare and have been described as blurred vision, amaurosis, mydriasis, miosis, enophthalmos, ophthalmoplegia, and diplopia. ${ }^{1}$ These cases have been described in dental practice with intraoral blockade of the inferior and superior alveolar nerves and have never been described as a potential sequela in relation to the TGN. Here, we present such a patient who suffered diplopia and sixth cranial nerve palsy following an extraoral maxillary nerve blockade.

\section{Case Report}

A 45-year-old man, a known case of TGN, presented to our pain clinic with complaints of sharp, lancinating pain over the left side of his face and jaw, corresponding to the distribution of the maxillary division of the left trigeminal nerve since past 5 years. There was no history of facial injury, dental surgeries, or intraoral infections. Physical and neurologic examination was unremarkable. Magnetic resonance imaging (MRI) revealed no evidence of neural compression by a vascular loop. His neuralgia was refractory to medical management with carbamazepine and pregabalin. Hence, maxillary nerve blockade was planned at the patient's request on an outpatient basis. His previous medical history and physical examination were unremarkable. Written informed consent was obtained. Inside the minor operating room (OR), the standard monitors were attached. Following sterile preparation and draping of the left side of the face, a $9.0-\mathrm{cm} 22 \mathrm{G}$ needle was introduced perpendicularly at the midpoint of the inferior border of the left zygomatic arch, to hit the lateral pterygoid plate. The needle was then withdrawn slightly and redirected anteriorly and superiorly. The needle was then carefully advanced up to $1 \mathrm{~cm}$ while stimulating the nerve at $1 \mathrm{~mA}$ for 0.1 second, until nerve stimulation was confirmed. After confirmation of negative aspiration, $2 \mathrm{~mL}$ of $2 \%$ lignocaine was injected, followed by $1 \mathrm{~mL}$ of absolute alcohol. The procedure was uneventful, and the patient had immediate and profound relief of pain.

A few seconds after the nerve block, the patient complained of discomfort in the ipsilateral eye and of double vision. On examination, the patient was unable to abduct the left eye, suggesting a palsy of the left abducens (CN VI) nerve. received

May 17, 2018

accepted after revision

May 24, 2018

published online

September 7, 2018
DOI https://doi.org/

$10.1055 / \mathrm{s}-0038-1665546$

ISSN 2348-0548.
Copyright @2018 Indian Society of Neuroanaesthesiology and Critical Care
License terms

(이 (1) $\Theta \circledast$ 
The eyelid had a slight droop (ptosis). All other eye movements were normal. There was no noticeable blanching of skin or numbness of upper face. Subsequent evaluation by a neurologist and an ophthalmologist was unremarkable, and the patient was reassured and managed conservatively. His symptoms resolved slowly over a period of 2 months, and the patient reported that his diplopia had ceased and that his vision had become normal.

\section{Discussion}

Similar cases of lateral rectus palsy have been described in dental literature, with both superior and inferior alveolar nerve blocks., ${ }^{2,3}$ However, to the best of our knowledge, this complication has not been reported with extraoral blockade of the maxillary branch of the trigeminal nerve. In this case, a couple of anatomical reasons may be offered. First, accidental puncture and injection of anesthetic agents into the middle meningeal artery remains the most likely explanation. The middle meningeal artery is a branch of the maxillary artery and enters the skull via the foramen spinosum. It gives off several branches within the middle cranial fossa, and branches back to the lacrimal and ophthalmic arteries. There is a possibility of retrograde flow of the anesthetic to the eye when the solution is injected under pressure, with a reduction in arterial pressure gradient during diastole. ${ }^{1}$ Second, accidental intravenous injection of anesthetics may cause flow toward the cavernous sinus via the pterygoid plexus and emissary veins. The abducens nerve runs through the cavernous sinus, and the oculomotor, trochlear, and ophthalmic and maxillary divisions of the trigeminal nerve lie within its lateral wall, which explains the occurrence of ophthalmological complications. ${ }^{4}$ Owing to its location within the cavernous sinus, the abducens nerve may be most susceptible to the effects of intravascularly deposited anesthetic agents.

Though ophthalmical complications after maxillary nerve blockade are extremely rare, they are distressing events.
Previous articles suggested aspiration prior to deposition of anesthetic solutions. However, this case shows that such complications are possible despite all precautions being taken. If the patient reports diplopia, injection should be stopped immediately and patient examination to be followed. Eye movements, blindness, facial muscle movement, and blanching of skin should be assessed. The patient should be reassured that this is a transient event that should resolve as the action of the anesthetic agent ceases. The patient should be escorted home and advised against driving until normal sight returns. Ophthalmological and neurological consultations may be advisable if complications last longer than anticipated. In this case, the complications lasted for 2 months possibly due to use of absolute alcohol as the neurolytic agent.

To conclude, we reported a case of reversible abducens nerve palsy associated with extraoral maxillary blockade of the trigeminal nerve. This complication occurred despite precautions such as aspiration and confirmation of needle tip with stimulation. Knowledge of such unanticipated complication and its potential cause may help the physician in planning for an appropriate management protocol.

\section{Conflict of Interest}

None.

\section{References}

1 Williams JV, Williams LR, Colbert SD, Revington PJ. Amaurosis, ophthalmoplegia, ptosis, mydriasis and periorbital blanching following inferior alveolar nerve anaesthesia. Oral Maxillofac Surg 2011;15(1):67-70

2 Pragasm M, Managutti A. Diplopia with local anesthesia. Natl J Maxillofac Surg 2011;2(1):82-85

3 You TM. Diplopia after inferior alveolar nerve block: case report and related physiology. J Dent Anesth Pain Med 2015; 15(2):93-96

4 Boynes SG, Echeverria Z, Abdulwahab M. Ocular complications associated with local anesthesia administration in dentistry. Dent Clin North Am 2010;54(4):677-686 